\title{
Electrochemical reduction potential correlation of some insect antifeedant potent 2-phenothiazinyl chalcones
}

\author{
K. Ranganathan ${ }^{1}$, R. Suresh ${ }^{1}$, D. Kamalakkannan ${ }^{1}$, R. Arulkumaran ${ }^{1}$, \\ R. Sundararajan ${ }^{1}$, S. P. Sakthinathan ${ }^{1}$, S. Vijayakumar ${ }^{1}$, G. Vanangamudi ${ }^{1}$, \\ K. Thirumurthy ${ }^{2}$, P. Mayavel $^{2}$, G. Thirunarayanan ${ }^{2, *}$ \\ ${ }^{1}$ Post Graduate and Research Department of Chemistry, Government Arts College, \\ C-Mutlur-608102, Chidambaram, India. \\ ${ }^{2}$ Department of Chemistry, Annamalai University, Annamalainagar-608002, India \\ *E-mail address: drgtnarayanan@gmail.com
}

\begin{abstract}
A series containing twelve substituted styryl 2-phenothiazenyl ketones have been synthesized. The electrochemical potentials of carbonyl and vinyl groups were measured. These potentials are correlated with Hammett substituent constants, $\mathrm{F}$ and $\mathrm{R}$ parameters. From the results of statistical analyses, the effects of substituent on the group reduction potentials have been discussed. The insect antifeedant activities of these chalcones have been studied using $4^{\text {th }}$ instar larvae Achoea Janata $L$ with castor leaf discs.
\end{abstract}

Keywords:

2-Phenothiazenyl chalcones; Electrochemistry; Reduction potential; Correlation analysis; Insect antifeedant activities

\section{INTRODUCTION}

Redox potentials of organic molecules are very useful for studying electrochemical behavior such as oxidation, reduction and group transformation process. These processes are essential for conversion of one functional group into another by electron transfer. Electrochemical synthesis is another kind process for the study of properties of reactant and products [1]. Boykin and co-workers [2] have studied the dc polarographic redox potential of chalcones and they found to good correlation with sigma constants. Alston and Fry [3] investigates the reduction potential correlation with Hammett sigma $p$-constants for the chalcone system having substituents in both phenyl rings. These system performs for evaluation of Bekkum, Verkade and Wepster correlation with correlation parameters. Moraleda et al [4] have studied to found good correlation of $\alpha, \beta$-unsaturated carbonyl compounds with half-wave potential, LUMO-HOMO energies with Hammett $\sigma_{p}$ constants. Chalcones possess a wide range of biological activities such as antibacterial [5], antifungal [6], antiviral [7], antifeedant [8], anticancer, antimalarial [9], antituberclosis [10], antiAIDS [11], antiplosmodial [12], anti-aids [9] and antioxidant [13] activities.

These multipronged activities present in different chalcones are examined against respective strains. 
The insect antifeedant activities of these chalcones have been examined against $4^{\text {th }}$ instar larvae's. In agricultural field the insect antifeedant activities of the compounds are very important for control of insects on plant leafs.

There is no report available for the correlation of redox potential and current of $\mathrm{C}=\mathrm{C}$ and $\mathrm{CO}$ of substituted styryl 2-phenothiazenyl ketones with Hammett substituent constants, F and $\mathrm{R}$ parameters and insect antifeedant activities in literature in the past.

Hence the authors have taken efforts to study the electrochemical behavior and insect antifeedant activities of 2-phenothiazenyl chalcones through the correlation of redox potential with Hammett substituent constants, Field and Resonance parameters.

The insect antifeedant activities of all synthesized chalcones have been studied using $4^{\text {th }}$ instar larvae Achoea Janata $L$ with castor semilooper.

\section{EXPERIMENTAL}

\section{1. General}

All chemicals were procured from Sigma-Aldrich and Merck chemical company. The purities of these synthesized chalcones were checked with their physical constants. The physical constants and spectral data of unknown chalcones are presented in Table 1. The Infrared spectra of compounds were recorded in avatar 300 infrared spectrophotometer.

The nuclear magnetic spectra of compounds were recorded in Bruker AV 400 type nuclear magnetic spectrometer applying $400 \mathrm{MHz}$ frequency for recording $1 \mathrm{H}$ and $100 \mathrm{MHz}$ frequency for ${ }^{13} \mathrm{C}$ spectrum using $\mathrm{CDCl}_{3}$ as solvent and TMS is an internal standard. Mass spectra of compounds were recorded in Varian 500 GC-Mass spectrometer.

The Reduction potential of these chalcones was determined in $\mathrm{HCH}$ Instruments inc., Electrochemical Analyzer USA using platinum wire electrode in spectral grade methanol.

\section{2. Synthesis of substituted styryl 2-phenothiazinyl ketones}

All substituted styryl 2-phenothiazinyl ketones were synthesized by Green Ground Chemistry method reported in the literature [14].

Phenothiazine-2-methyl ketone $(0.02 \mathrm{mmol})$, substituted benzaldehydes $(0.02 \mathrm{mmol})$ and sodium hydroxide $(0.5 \mathrm{~g})$ in a mortar was ground with pestle for 20 minutes (Scheme 1). Completion of the reaction was confirmed by thin layer chromatography, the reaction mixture was poured into $25 \mathrm{ml}$ of cold water.

The obtained brown solid was separated by filtration. The pure brown chalcones were obtained by crystallization of these brown solid with ethanol. Physical constants, analytical, UV and mass spectral data were presented in (Table 1).

Infrared spectral data of all chalcones were given in (Table 2). The NMR data of synthesized styryl 2-phenothiazinyl ketones are tabulated in (Table 3). 


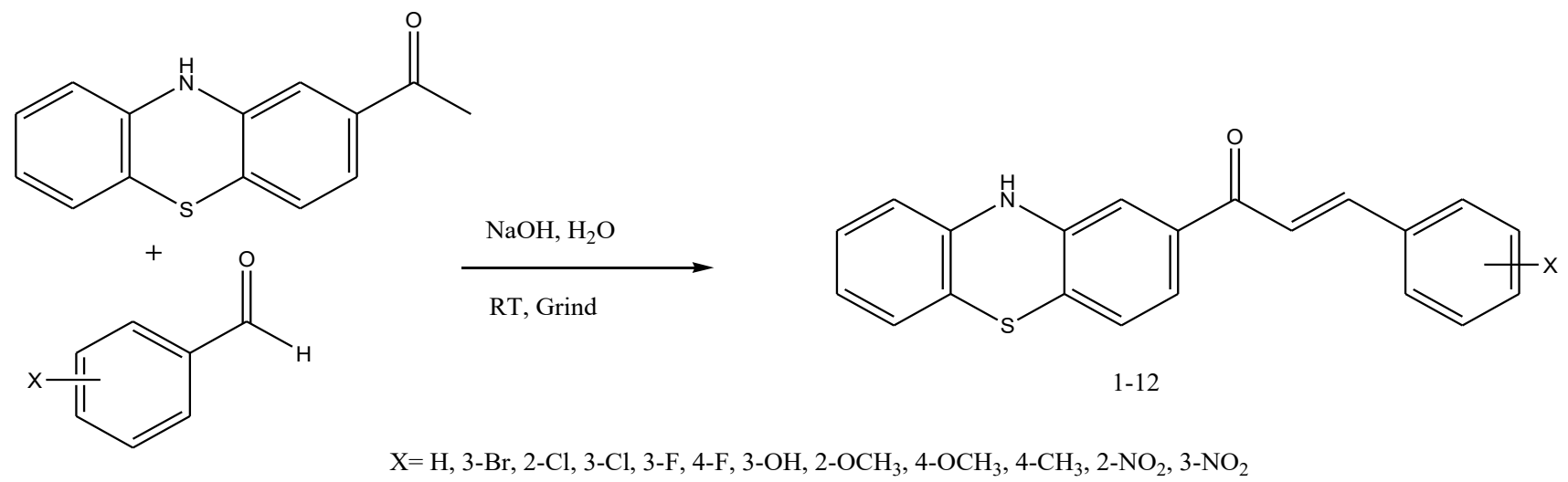

Scheme 1. Synthesis of 2-phenothiazinyl chalcones.

Table 1. Physical constants, analytical, Ultraviolet and mass spectral data of 2-phenothiazinyl chalcones

\begin{tabular}{|c|c|c|c|c|c|c|}
\hline Entry & $\mathbf{X}$ & Mol. Formula & m.p. ${ }^{\circ} \mathrm{C}$ & M.W & $\operatorname{UV\lambda } \max (\mathbf{n m})$ & $\operatorname{Mass}(\mathbf{m} / \mathbf{z})$ \\
\hline 1 & $\mathrm{H}$ & $\mathrm{C}_{21} \mathrm{H}_{15} \mathrm{NOS}$ & 170 & 329 & 286.20 & $\begin{array}{c}329\left[\mathrm{M}^{+}\right], 312,296,286,267,241, \\
226,198,171,165,154,148,131, \\
113,103,85,77,58\end{array}$ \\
\hline 2 & $3-\mathrm{Br}$ & $\mathrm{C}_{21} \mathrm{H}_{14} \mathrm{BrNOS}$ & 205 & 408 & 281.20 & $\begin{array}{c}408\left[\mathrm{M}^{+}\right], 410[\mathrm{M}+2], 390,376,328, \\
310,299,281,267,241,226,211, \\
148,182,171,164,148,127,113, \\
102,97,73,58\end{array}$ \\
\hline 3 & $2-\mathrm{Cl}$ & $\mathrm{C}_{21} \mathrm{H}_{14} \mathrm{ClNOS}$ & 338(D.P) & 363 & 299.00 & $\begin{array}{c}363\left[\mathrm{M}^{+}\right], 365[\mathrm{M}+2], 328,310, \\
296,286,267,254,241,226,211,1 \\
98,183,164,148,139,113,97,77, \\
58\end{array}$ \\
\hline 4 & $3-\mathrm{Cl}$ & $\mathrm{C}_{21} \mathrm{H}_{14} \mathrm{ClNOS}$ & 143 & 363 & 307.20 & $\begin{array}{c}363\left[\mathrm{M}^{+}\right], 365[\mathrm{M}+2], 328,310, \\
296,286,267,254,241,226,211, \\
198,183,164,148,139,113,97,77, \\
58\end{array}$ \\
\hline 5 & $3-\mathrm{F}$ & $\mathrm{C}_{21} \mathrm{H}_{14} \mathrm{FNOS}$ & 201 & 347 & 308.00 & $\begin{array}{c}347\left[\mathrm{M}^{+}\right], 347[\mathrm{M}+2], 330,314 \\
285,267,252,226,198,173,154 \\
149,121,101,97,73,58\end{array}$ \\
\hline 6 & $4-\mathrm{F}$ & $\mathrm{C}_{21} \mathrm{H}_{14} \mathrm{FNOS}$ & 143 & 347 & 314.60 & $\begin{array}{c}347\left[\mathrm{M}^{+}\right], 347[\mathrm{M}+2], 330,314 \\
285,267,252,226,198,173,154, \\
149,121,101,97,73,58\end{array}$ \\
\hline 7 & $3-\mathrm{OH}$ & $\mathrm{C}_{21} \mathrm{H}_{15} \mathrm{NO}_{2} \mathrm{~S}$ & 128 & 345 & 280.80 & $\begin{array}{c}345\left[\mathrm{M}^{+}\right], 327,313,295,281,267 \\
251,241,226,221,207,198,193 \\
169,154,135,113,99,73,69\end{array}$ \\
\hline 8 & $2-\mathrm{OCH}_{3}$ & $\mathrm{C}_{22} \mathrm{H}_{17} \mathrm{NO}_{2} \mathrm{~S}$ & 156 & 359 & 342.00 & $\begin{array}{c}359\left[\mathrm{M}^{+}\right], 344,326,316,298,283 \\
267,254,241,223,198,183,179 \\
161,133,118,97,73,58\end{array}$ \\
\hline
\end{tabular}




\begin{tabular}{|c|c|c|c|c|c|l|}
\hline $\mathbf{9}$ & $4-\mathrm{OCH}_{3}$ & $\mathrm{C}_{22} \mathrm{H}_{17} \mathrm{NO}_{2} \mathrm{~S}$ & 162 & 359 & 341.20 & $\begin{array}{l}359\left[\mathrm{M}^{+}\right], 344,326,316,298,283, \\
267,254,241,223,198,183,179, \\
161,133,118,97,73,58\end{array}$ \\
\hline $\mathbf{1 0}$ & $4-\mathrm{CH}_{3}$ & $\mathrm{C}_{22} \mathrm{H}_{17} \mathrm{NOS}$ & 356 (D.P.) & 343 & 323.40 & $\begin{array}{l}343\left[\mathrm{M}^{+}\right], 328,310,300,281,267, \\
252,241,223,198,164,145,127, \\
115,102,91,77,65\end{array}$ \\
\hline $\mathbf{1 1}$ & $2-\mathrm{NO}_{2}$ & $\mathrm{C}_{21} \mathrm{H}_{14} \mathrm{~N}_{2} \mathrm{O}_{3} \mathrm{~S}$ & 324 (D.P.) & 374 & 281.80 & $\begin{array}{l}374\left[\mathrm{M}^{+}\right], 358,311,299,286,267, \\
254,241,223,198,154,127,121, \\
102,89,77,65\end{array}$ \\
\hline $\mathbf{1 2}$ & $3-\mathrm{NO}_{2}$ & $\mathrm{C}_{21} \mathrm{H}_{14} \mathrm{~N}_{2} \mathrm{O}_{3} \mathrm{~S}$ & 334 (D.P.) & 374 & 298.00 & $\begin{array}{l}374\left[\mathrm{M}^{+}\right], 358,342,311,299,286,254,241,223,198,171,154, \\
267,254,121,102,89,77,65\end{array}$ \\
\hline
\end{tabular}

Table 2. Infrared spectral data $v\left(\mathrm{~cm}^{-1}\right)$ of 2-phenothiazinyl chalcones.

\begin{tabular}{|c|c|c|c|c|c|c|c|}
\hline Entry & $\mathbf{X}$ & COs-cis & COs-trans & CHip & CHop & CH=CHop & $>$ C $=$ C $<$ op \\
\hline 1 & $\mathrm{H}$ & 1660.60 & 1627.80 & 1155.30 & 736.80 & 1078.10 & 680.80 \\
\hline 2 & $3-\mathrm{Br}$ & 1664.50 & 1627.80 & 1155.30 & 740.60 & 1093.60 & 638.40 \\
\hline 3 & $2-\mathrm{Cl}$ & 1656.70 & 1618.20 & 1178.40 & 752.20 & 1041.50 & 588.20 \\
\hline 4 & $3-\mathrm{Cl}$ & 1649.00 & 1587.30 & 1191.90 & 734.80 & 1080.10 & 582.50 \\
\hline 5 & $3-\mathrm{F}$ & 1651.00 & 1585.40 & 1186.10 & 777.30 & 1082.00 & 584.40 \\
\hline 6 & $4-\mathrm{F}$ & 1652.90 & 1589.20 & 1184.20 & 736.80 & 1083.90 & 563.20 \\
\hline 7 & $3-\mathrm{OH}$ & 1664.50 & 1635.50 & 1157.20 & 740.60 & 1093.60 & 638.40 \\
\hline 8 & $2-\mathrm{OCH}_{3}$ & 1652.90 & 1591.20 & 1172.60 & 740.60 & 1080.10 & 576.70 \\
\hline 9 & $4-\mathrm{OCH}_{3}$ & 1651.00 & 1587.30 & 1153.40 & 740.60 & 1082.00 & 582.50 \\
\hline 10 & $4-\mathrm{CH}_{3}$ & 1652.90 & 1627.80 & 1184.20 & 798.50 & 1083.90 & 565.10 \\
\hline 11 & $2-\mathrm{NO}_{2}$ & 1658.70 & 1598.90 & 1188.10 & 786.90 & 1083.90 & 565.10 \\
\hline 12 & $3-\mathrm{NO}_{2}$ & 1658.70 & 1588.30 & 1189.10 & 801.50 & 1084.00 & 588.30 \\
\hline
\end{tabular}

Table 3. The NMR spectral chemical shift $\delta(\mathrm{ppm})$ of 2-phenothiazinyl chalcones.

\begin{tabular}{|c|c|c|c|c|c|c|c|c|}
\hline \multirow[b]{2}{*}{ Entry } & \multirow[b]{2}{*}{$\mathbf{X}$} & \multicolumn{3}{|c|}{${ }^{1}$ H NMR } & \multicolumn{4}{|c|}{${ }^{13} \mathrm{C}$ NMR } \\
\hline & & $\begin{array}{c}\delta \mathbf{H \alpha} \\
(1 \mathbf{H}, d)\end{array}$ & $\begin{array}{c}\delta \mathbf{H} \beta \\
(1 \mathbf{H}, d)\end{array}$ & Substt. & $\delta \mathrm{CO}$ & $\delta \mathrm{Ca}$ & $\delta \mathrm{C} \beta$ & Substt. \\
\hline 1 & $\mathrm{H}$ & 7.489 & 7.845 & --- & 188.79 & 121.89 & 145.16 & --- \\
\hline 2 & $3-\mathrm{Br}$ & 6.693 & 6.932 & --- & 196.76 & 123.20 & 141.99 & --- \\
\hline 3 & $2-\mathrm{Cl}$ & 7.847 & 8.069 & --- & 187.69 & 122.07 & 143.74 & --- \\
\hline 4 & $3-\mathrm{Cl}$ & 7.678 & 7.882 & --- & 187.85 & 122.10 & 142.14 & --- \\
\hline 5 & $3-\mathrm{F}$ & 7.656 & 7.890 & --- & 187.89 & 122.00 & 141.14 & --- \\
\hline 6 & $4-\mathrm{F}$ & 7.045 & 7.758 & --- & 187.90 & 122.04 & 142.46 & --- \\
\hline 7 & $3-\mathrm{OH}$ & 6.681 & 6.926 & $\begin{array}{c}3.336 \\
(1 \mathrm{H}, s) \\
\end{array}$ & 196.79 & 123.23 & 142.01 & --- \\
\hline 8 & $2-\mathrm{OCH}_{3}$ & 6.708 & 6.955 & $\begin{array}{c}3.819 \\
(3 \mathrm{H}, s)\end{array}$ & 187.82 & 119.29 & 143.77 & $\begin{array}{c}55.36 \\
\left(\mathrm{OCH}_{3}\right)\end{array}$ \\
\hline 9 & $4-\mathrm{OCH}_{3}$ & 7.053 & 7.633 & $\begin{array}{c}3.820 \\
(3 \mathrm{H}, s)\end{array}$ & 187.95 & 122.05 & 142.09 & $\begin{array}{c}55.37 \\
\left(\mathrm{OCH}_{3}\right)\end{array}$ \\
\hline 10 & $4-\mathrm{CH}_{3}$ & 6.956 & 7.675 & $\begin{array}{c}2.355 \\
(3 \mathrm{H}, s) \\
\end{array}$ & 187.92 & 120.76 & 143.78 & $\begin{array}{l}21.03 \\
\left(\mathrm{CH}_{3}\right) \\
\end{array}$ \\
\hline 11 & $2-\mathrm{NO}_{2}$ & 7.778 & 7.961 & --- & 187.67 & 122.78 & 142.16 & --- \\
\hline 12 & $3-\mathrm{NO}_{2}$ & 8.108 & 8.874 & --- & 188.86 & 122.89 & 142.39 & --- \\
\hline
\end{tabular}




\section{RESULTS AND DISCUSSION}

In the present study the ketones were chosen as an $\alpha, \beta$-unsaturated system. These system exhibit as $E$ stable conformers. The measured reduction potential (v) and the corresponding current (i) are presented in (Table 4). These potential and currents are correlated with Hammett substituent constants, $\mathrm{F}$ and $\mathrm{R}$ parameters using single and multi-regression analyses [15-17]. The results of statistical analysis of reduction potential (v) and the corresponding current (i) with Hammett substituent constants, F and $\mathrm{R}$ parameters are presented in (Table 5). From Table 5, correlation of reduction potential of $\mathrm{C}=\mathrm{C}$ with sigma constants, $\mathrm{F}$ and $\mathrm{R}$ parameters were fail. On the other hand this current (i) were satisfactory correlated with sigma constants. The polar, resonance and fields were failing in correlation with these parameters. This is due to the inability of effect of substituents

Table 4. The reduction potential (v) and current (i) of carbonyl and $\mathrm{C}=\mathrm{C}$ groups of 2-phenothiazinyl chalcones.

\begin{tabular}{|c|c|c|c|c|c|}
\hline S. No. & Entry & $\begin{array}{c}\text { Current(i) } \\
\mathbf{C = C}\end{array}$ & $\begin{array}{c}\text { Current(i) } \\
\mathbf{C}=\mathbf{O}\end{array}$ & $\begin{array}{c}\text { Potential(v) } \\
\mathbf{C}=\mathbf{C}\end{array}$ & $\begin{array}{c}\text { Potential(v) } \\
\mathbf{C}=\mathbf{O}\end{array}$ \\
\hline 1 & $\mathrm{H}$ & -0.02 & 4.936 & 0.123 & 0.243 \\
\hline 2 & $3-\mathrm{Br}$ & -2.46 & 9.415 & 0.321 & 0.881 \\
\hline 3 & $2-\mathrm{Cl}$ & 1.602 & -2.606 & 1.373 & -1.025 \\
\hline 4 & $3-\mathrm{Cl}$ & -4.088 & -1.430 & 0.403 & -0.181 \\
\hline 5 & $3-\mathrm{F}$ & -2.193 & -4.955 & 0.369 & -0.340 \\
\hline 6 & $4-\mathrm{F}$ & -1.532 & -5.935 & 0.385 & -0.097 \\
\hline 7 & $3-\mathrm{OH}$ & 3.688 & -5.376 & 0.365 & -0.320 \\
\hline 8 & $2-\mathrm{OCH}_{3}$ & -3.496 & -4.145 & 0.271 & -0.575 \\
\hline 9 & $4-\mathrm{OCH}_{3}$ & -4.238 & -2.246 & -0.604 & -0.423 \\
\hline 10 & $4-\mathrm{CH}_{3}$ & -7.032 & -1.969 & -0.558 & -1.314 \\
\hline 11 & $2-\mathrm{NO}_{2}$ & -6.212 & -3.531 & -0.634 & -1.610 \\
\hline 12 & $3-\mathrm{NO}_{2}$ & 0.283 & 1.954 & -0.296 & -3.455 \\
\hline
\end{tabular}

not obeyed the potential and current and the conjugation between the substituent and carbonyl group shown in Figure 1. All correlation gave negative $\rho$ values except $\sigma_{I}$ with reduction potential of $\mathrm{C}=\mathrm{C}$ and vice versa with $\mathrm{C}=\mathrm{C}$ current. All correlations are fail with the reduction potential of $\mathrm{CO}$ with sigma constants, $\mathrm{F}$ and $\mathrm{R}$ parameters. A satisfactory correlation obtained for $\mathrm{R}$ parameter with reduction current of CO. Remaining sigma constants and Field parameters fail in correlations. This failure was due to conjugative structure shown in Figure 1.<smiles>CO[C+]=c1ccc(=C/C=C(\[O-])c2ccc3c(c2)Nc2ccccc2S3)cc1</smiles>

Figure 1. Resonance-conjugative structure 
Table 5. Results of statistical analysis of reduction potential(v), current(i) of $\mathrm{C}=\mathrm{C}$ and $\mathrm{CO}$ of substituted styryl 2-phenothiazenyl ketones with Hammett substituent constants $\sigma, \sigma^{+}, \sigma_{\mathrm{I}}, \sigma_{\mathrm{R}}$ and $\mathrm{F}$ and $\mathrm{R}$ parameters.

\begin{tabular}{|c|c|c|c|c|c|c|c|}
\hline Frequency & $\begin{array}{l}\text { Cons- } \\
\text { tants }\end{array}$ & $\mathbf{r}$ & $\mathbf{I}$ & $\rho$ & $\mathbf{s}$ & $\mathbf{n}$ & Correlated derivatives \\
\hline \multirow{6}{*}{$\mathrm{C}=\mathrm{C}(\mathrm{v})$} & $\sigma$ & 0.812 & -0.398 & -0.250 & 0.63 & 12 & $\begin{array}{l}\mathrm{H}, 3-\mathrm{Br}, 2-\mathrm{Cl}, 3-\mathrm{Cl}, 3-\mathrm{F}, 4-\mathrm{F}, 3-\mathrm{OH}, 2- \\
\mathrm{OCH}_{3}, 4-\mathrm{OCH}_{3}, 4-\mathrm{CH}_{3}, 2-\mathrm{NO}_{2}, 3-\mathrm{NO}_{2}\end{array}$ \\
\hline & $\sigma^{+}$ & 0.811 & -0.421 & -0.146 & 0.63 & 12 & $\begin{array}{l}\mathrm{H}, 3-\mathrm{Br}, 2-\mathrm{Cl}, 3-\mathrm{Cl}, 3-\mathrm{F}, 4-\mathrm{F}, 3-\mathrm{OH}, 2- \\
\mathrm{OCH}_{3}, 4-\mathrm{OCH}_{3}, 4-\mathrm{CH}_{3}, 2-\mathrm{NO}_{2}, 3-\mathrm{NO}_{2}\end{array}$ \\
\hline & $\sigma_{\mathrm{I}}$ & 0.821 & -0.292 & -0.375 & 0.63 & 12 & $\begin{array}{l}\mathrm{H}, 3-\mathrm{Br}, 2-\mathrm{Cl}, 3-\mathrm{Cl}, 3-\mathrm{F}, 4-\mathrm{F}, 3-\mathrm{OH}, 2- \\
\mathrm{OCH}_{3}, 4-\mathrm{OCH}_{3}, 4-\mathrm{CH}_{3}, 2-\mathrm{NO}_{2}, 3-\mathrm{NO}_{2}\end{array}$ \\
\hline & $\sigma_{\mathrm{R}}$ & 0.828 & -0.667 & -0.837 & 0.61 & 12 & $\begin{array}{l}\mathrm{H}, 3-\mathrm{Br}, 2-\mathrm{Cl}, 3-\mathrm{Cl}, 3-\mathrm{F}, 4-\mathrm{F}, 3-\mathrm{OH}, 2- \\
\mathrm{OCH}_{3}, 4-\mathrm{OCH}_{3}, 4-\mathrm{CH}_{3}, 2-\mathrm{NO}_{2}, 3-\mathrm{NO}_{2}\end{array}$ \\
\hline & $\mathrm{F}$ & 0.803 & -0.387 & -0.098 & 0.64 & 12 & $\begin{array}{l}\mathrm{H}, 3-\mathrm{Br}, 2-\mathrm{Cl}, 3-\mathrm{Cl}, 3-\mathrm{F}, 4-\mathrm{F}, 3-\mathrm{OH}, 2- \\
\mathrm{OCH}_{3}, 4-\mathrm{OCH}_{3}, 4-\mathrm{CH}_{3}, 2-\mathrm{NO}_{2}, 3-\mathrm{NO}_{2}\end{array}$ \\
\hline & $\mathrm{R}$ & 0.822 & -0.889 & -0.414 & 0.62 & 12 & $\begin{array}{l}\mathrm{H}, 3-\mathrm{Br}, 2-\mathrm{Cl}, 3-\mathrm{Cl}, 3-\mathrm{F}, 4-\mathrm{F}, 3-\mathrm{OH}, 2- \\
\mathrm{OCH}_{3}, 4-\mathrm{OCH}_{3}, 4-\mathrm{CH}_{3}, 2-\mathrm{NO}_{2}, 3-\mathrm{NO}_{2}\end{array}$ \\
\hline \multirow[t]{6}{*}{$\mathrm{C}=\mathrm{C}(\mathrm{i})$} & $\sigma$ & 0.907 & -0.729 & -1.956 & 3.00 & 12 & $\begin{array}{l}\mathrm{H}, 3-\mathrm{Br}, 2-\mathrm{Cl}, 3-\mathrm{Cl}, 3-\mathrm{F}, 4-\mathrm{F}, 3-\mathrm{OH}, 2- \\
\mathrm{OCH}_{3}, 4-\mathrm{OCH}_{3}, 4-\mathrm{CH}_{3}, 2-\mathrm{NO}_{2}, 3-\mathrm{NO}_{2}\end{array}$ \\
\hline & $\sigma^{+}$ & 0.801 & -0.608 & -2.020 & 3.00 & 12 & $\begin{array}{l}\mathrm{H}, 3-\mathrm{Br}, 2-\mathrm{Cl}, 3-\mathrm{Cl}, 3-\mathrm{F}, 4-\mathrm{F}, 3-\mathrm{OH}, 2- \\
\mathrm{OCH}_{3}, 4-\mathrm{OCH}_{3}, 4-\mathrm{CH}_{3}, 2-\mathrm{NO}_{2}, 3-\mathrm{NO}_{2}\end{array}$ \\
\hline & $\sigma_{\mathrm{I}}$ & 0.802 & 0.298 & -2.142 & 3.01 & 12 & $\begin{array}{l}\mathrm{H}, 3-\mathrm{Br}, 2-\mathrm{Cl}, 3-\mathrm{Cl}, 3-\mathrm{F}, 4-\mathrm{F}, 3-\mathrm{OH}, 2- \\
\mathrm{OCH}_{3}, 4-\mathrm{OCH}_{3}, 4-\mathrm{CH}_{3}, 2-\mathrm{NO}_{2}, 3-\mathrm{NO}_{2}\end{array}$ \\
\hline & $\sigma_{\mathrm{R}}$ & 0.819 & -2.719 & -2.802 & 2.95 & 12 & $\begin{array}{l}\mathrm{H}, 3-\mathrm{Br}, 2-\mathrm{Cl}, 3-\mathrm{Cl}, 3-\mathrm{F}, 4-\mathrm{F}, 3-\mathrm{OH}, 2- \\
\mathrm{OCH}_{3}, 4-\mathrm{OCH}_{3}, 4-\mathrm{CH}_{3}, 2-\mathrm{NO}_{2}, 3-\mathrm{NO}_{2}\end{array}$ \\
\hline & $\mathrm{F}$ & 0.714 & 0.169 & -2.954 & 4.89 & 12 & $\begin{array}{l}\mathrm{H}, 3-\mathrm{Br}, 2-\mathrm{Cl}, 3-\mathrm{Cl}, 3-\mathrm{F}, 4-\mathrm{F}, 3-\mathrm{OH}, 2- \\
\mathrm{OCH}_{3}, 4-\mathrm{OCH}_{3}, 4-\mathrm{CH}_{3}, 2-\mathrm{NO}_{2}, 3-\mathrm{NO}_{2}\end{array}$ \\
\hline & $\mathrm{R}$ & 0.813 & -3.919 & -1.782 & 3.18 & 12 & $\begin{array}{l}\mathrm{H}, 3-\mathrm{Br}, 2-\mathrm{Cl}, 3-\mathrm{Cl}, 3-\mathrm{F}, 4-\mathrm{F}, 3-\mathrm{OH}, 2- \\
\mathrm{OCH}_{3}, 4-\mathrm{OCH}_{3}, 4-\mathrm{CH}_{3}, 2-\mathrm{NO}_{2}, 3-\mathrm{NO}_{2}\end{array}$ \\
\hline \multirow[t]{6}{*}{$\mathrm{C}=\mathrm{O}(\mathrm{v})$} & $\sigma$ & 0.829 & -0.577 & -0.430 & 0.75 & 12 & $\begin{array}{l}\mathrm{H}, 3-\mathrm{Br}, 2-\mathrm{Cl}, 3-\mathrm{Cl}, 3-\mathrm{F}, 4-\mathrm{F}, 3-\mathrm{OH}, 2- \\
\mathrm{OCH}_{3}, 4-\mathrm{OCH}_{3}, 4-\mathrm{CH}_{3}, 2-\mathrm{NO}_{2}, 3-\mathrm{NO}_{2}\end{array}$ \\
\hline & $\sigma^{+}$ & 0.823 & -0.313 & -0.516 & 0.76 & 12 & $\begin{array}{l}\mathrm{H}, 3-\mathrm{Br}, 2-\mathrm{Cl}, 3-\mathrm{Cl}, 3-\mathrm{F}, 4-\mathrm{F}, 3-\mathrm{OH}, 2- \\
\mathrm{OCH}_{3}, 4-\mathrm{OCH}_{3}, 4-\mathrm{CH}_{3}, 2-\mathrm{NO}_{2}, 3-\mathrm{NO}_{2}\end{array}$ \\
\hline & $\sigma_{\mathrm{I}}$ & 0.823 & -0.751 & -0.238 & 0.76 & 12 & $\begin{array}{l}\mathrm{H}, 3-\mathrm{Br}, 2-\mathrm{Cl}, 3-\mathrm{Cl}, 3-\mathrm{F}, 4-\mathrm{F}, 3-\mathrm{OH}, 2- \\
\mathrm{OCH}_{3}, 4-\mathrm{OCH}_{3}, 4-\mathrm{CH}_{3}, 2-\mathrm{NO}_{2}, 3-\mathrm{NO}_{2}\end{array}$ \\
\hline & $\sigma_{\mathrm{R}}$ & 0.839 & -1.293 & -0.838 & 0.72 & 12 & $\begin{array}{l}\mathrm{H}, 3-\mathrm{Br}, 2-\mathrm{Cl}, 3-\mathrm{Cl}, 3-\mathrm{F}, 4-\mathrm{F}, 3-\mathrm{OH}, 2- \\
\mathrm{OCH}_{3}, 4-\mathrm{OCH}_{3}, 4-\mathrm{CH}_{3}, 2-\mathrm{NO}_{2}, 3-\mathrm{NO}_{2}\end{array}$ \\
\hline & $\mathrm{F}$ & 0.813 & -0.388 & -0.369 & 0.77 & 12 & $\begin{array}{l}\mathrm{H}, 3-\mathrm{Br}, 2-\mathrm{Cl}, 3-\mathrm{Cl}, 3-\mathrm{F}, 4-\mathrm{F}, 3-\mathrm{OH}, 2- \\
\mathrm{OCH}_{3}, 4-\mathrm{OCH}_{3}, 4-\mathrm{CH}_{3}, 2-\mathrm{NO}_{2}, 3-\mathrm{NO}_{2}\end{array}$ \\
\hline & $\mathrm{R}$ & 0.833 & -0.636 & -0.750 & 0.74 & 12 & $\begin{array}{l}\mathrm{H}, 3-\mathrm{Br}, 2-\mathrm{Cl}, 3-\mathrm{Cl}, 3-\mathrm{F}, 4-\mathrm{F}, 3-\mathrm{OH}, 2- \\
\mathrm{OCH}_{3}, 4-\mathrm{OCH}_{3}, 4-\mathrm{CH}_{3}, 2-\mathrm{NO}_{2}, 3-\mathrm{NO}_{2}\end{array}$ \\
\hline \multirow[t]{6}{*}{$\mathrm{C}=\mathrm{O}$ (i) } & $\sigma$ & 0.808 & 0.158 & 0.251 & 0.57 & 12 & $\begin{array}{l}\mathrm{H}, 3-\mathrm{Br}, 2-\mathrm{Cl}, 3-\mathrm{Cl}, 3-\mathrm{F}, 4-\mathrm{F}, 3-\mathrm{OH}, 2- \\
\mathrm{OCH}_{3}, 4-\mathrm{OCH}_{3}, 4-\mathrm{CH}_{3}, 2-\mathrm{NO}_{2}, 3-\mathrm{NO}_{2}\end{array}$ \\
\hline & $\sigma^{+}$ & 0.821 & 0.316 & 0.260 & 0.55 & 12 & $\begin{array}{l}\mathrm{H}, 3-\mathrm{Br}, 2-\mathrm{Cl}, 3-\mathrm{Cl}, 3-\mathrm{F}, 4-\mathrm{F}, 3-\mathrm{OH}, 2- \\
\mathrm{OCH}_{3}, 4-\mathrm{OCH}_{3}, 4-\mathrm{CH}_{3}, 2-\mathrm{NO}_{2}, 3-\mathrm{NO}_{2}\end{array}$ \\
\hline & $\sigma_{\mathrm{I}}$ & 0.829 & 0.818 & -0.021 & 0.55 & 12 & $\begin{array}{l}\mathrm{H}, 3-\mathrm{Br}, 2-\mathrm{Cl}, 3-\mathrm{Cl}, 3-\mathrm{F}, 4-\mathrm{F}, 3-\mathrm{OH}, 2- \\
\mathrm{OCH}_{3}, 4-\mathrm{OCH}_{3}, 4-\mathrm{CH}_{3}, 2-\mathrm{NO}_{2}, 3-\mathrm{NO}_{2}\end{array}$ \\
\hline & $\sigma_{\mathrm{R}}$ & 0.718 & -0.495 & 0.125 & 0.56 & 12 & $\begin{array}{l}\mathrm{H}, 3-\mathrm{Br}, 2-\mathrm{Cl}, 3-\mathrm{Cl}, 3-\mathrm{F}, 4-\mathrm{F}, 3-\mathrm{OH}, 2- \\
\mathrm{OCH}_{3}, 4-\mathrm{OCH}_{3}, 4-\mathrm{CH}_{3}, 2-\mathrm{NO}_{2}, 3-\mathrm{NO}_{2}\end{array}$ \\
\hline & $\mathrm{F}$ & 0.822 & 0.859 & 0.051 & 0.56 & 12 & $\begin{array}{l}\mathrm{H}, 3-\mathrm{Br}, 2-\mathrm{Cl}, 3-\mathrm{Cl}, 3-\mathrm{F}, 4-\mathrm{F}, 3-\mathrm{OH}, 2- \\
\mathrm{OCH}_{3}, 4-\mathrm{OCH}_{3}, 4-\mathrm{CH}_{3}, 2-\mathrm{NO}_{2}, 3-\mathrm{NO}_{2}\end{array}$ \\
\hline & $\mathrm{R}$ & 0.911 & -0.192 & 0.192 & 0.57 & 12 & $\begin{array}{l}\mathrm{H}, 3-\mathrm{Br}, 2-\mathrm{Cl}, 3-\mathrm{Cl}, 3-\mathrm{F}, 4-\mathrm{F}, 3-\mathrm{OH}, 2- \\
\mathrm{OCH}_{3}, 4-\mathrm{OCH}_{3}, 4-\mathrm{CH}_{3}, 2-\mathrm{NO}_{2}, 3-\mathrm{NO}_{2}\end{array}$ \\
\hline
\end{tabular}

$\mathrm{r}=$ Correlation coefficient; $\rho=$ Slope; $\mathrm{I}=$ Intercept; $\mathrm{s}=$ Standard deviation; $\mathrm{n}=$ Number of substituents. 
Some of the single parameter electrochemical correlations were fails with Hammett sigma constants, $\mathrm{F}$ and $\mathrm{R}$ parameters. On the other hand the multi-regressions were worthwhile and leads to satisfactory correlated with Swain-Lupton [18] F and R parameters collectively. The correlation equations $1-8$ are as

$$
\begin{gathered}
\mathrm{C}=\mathrm{C}(\mathrm{v})=-0.540( \pm 0.042)-0.349( \pm 0.081) \sigma_{\mathrm{I}}-0.827( \pm 0.081) \sigma_{\mathrm{R}} \\
(\mathrm{R}=0.930, \mathrm{n}=12, \mathrm{P}>90 \%) \\
\mathrm{C}=\mathrm{C}(\mathrm{v})=0.534( \pm 0.039)-0.142( \pm 0.078) \mathrm{F}-0.420( \pm 0.050) \mathrm{R} \\
(R=0.923, \mathrm{n}=12, \mathrm{P}>90 \%) \\
\mathrm{C}=\mathrm{C}(\mathrm{i})=1.622( \pm 0.291)-3.546( \pm 0.593) \sigma_{\mathrm{I}}+11.362( \pm 5.682) \sigma_{\mathrm{R}} \\
(R=0.951, \mathrm{n}=12, \mathrm{P}>95 \%) \\
\mathrm{C}=\mathrm{C}(\mathrm{i})=1.282( \pm 0.267)-4.413( \pm 0.051) \mathrm{F}+6.271( \pm 0.141) \mathrm{R} \\
(R=0.950, \mathrm{n}=12, \mathrm{P}>95 \%) \\
\mathrm{C}=\mathrm{O}(\mathrm{v})=-0.565( \pm 0.049)-0.683( \pm 0.096) \sigma_{\mathrm{I}}-1.243( \pm 0.097) \sigma_{\mathrm{R}} \\
(R=0.944, \mathrm{n}=12, \mathrm{P}>90 \%) \\
\mathrm{C}=\mathrm{O}(\mathrm{v})=-0.588( \pm 0.048)-0.387( \pm 0.090) \mathrm{F}-0.636( \pm 0.059) \mathrm{R} \\
(R=0.935, \mathrm{n}=12, \mathrm{P}>90 \%) \\
\mathrm{C}=\mathrm{O}(\mathrm{i})=-0.177( \pm 0.370)+0.834( \pm 0.076) \sigma_{\mathrm{I}}-0.591( \pm 0.071) \sigma_{\mathrm{R}} \\
(R=0.935, \mathrm{n}=12, \mathrm{P}>90 \%) \\
\mathrm{C}=\mathrm{O}(\mathrm{i})=-0.009( \pm 0.003)+0.542( \pm 0.075) \mathrm{F}-0.170( \pm 0.045) \mathrm{R} \\
(R=0.958, \mathrm{n}=12, \mathrm{P}>95 \%)
\end{gathered}
$$

\section{1. Insect antifeedant activity}

The multipronged activities present in different epoxy ketones are intended to examine their insect antifeedant activities against castor semilooper. The larvae of Achoea Janata L were reared as described on the leaves of castor Riclnus communls in the laboratory at the temperature range of $26{ }^{\circ} \mathrm{C} \pm 1{ }^{\circ} \mathrm{C}$ and a relative humidity of $75-85 \%$. The leaf - disc bioassay method $[8,19]$ was used against the $4^{\text {th }}$ instar larvae to measure the antifeedant activity. The $4^{\text {th }}$ instar larvae were selected for testing because the larvae at this stage feed very voraciously.

\section{2. Measurement of insect antifeedant activity of chalcones}

Leaf discs of a diameter of $1.85 \mathrm{~cm}$ were punched from castor leaves with the petioles intact. All chalcones. Insect antifeedant activities of substituted styryl 2-phenothiazinyl ketones were dissolved in acetone at a concentration of 200 ppm dipped for 5 minutes. The leaf discs were airdried and placed in one litre beaker containing little water in order to facilitate translocation of water. Therefore the leaf discs remains fresh throughout the duration of the rest, $4^{\text {th }}$ instar larvae of the test insect, which had been preserved on the leaf discs of all chalcones and allowed to feed on them for 24 hours. The areas of the leaf discs consumed were measured by Dethlers [19] method. The observed antifeedant activity of chalcones was presented in (Table 6). 
Table 6. Insect antifeedant activities of substituted styryl 2-phenothiazenyl ketones.

\begin{tabular}{|c|c|c|c|c|c|c|c|c|c|c|c|}
\hline$\underset{\Xi}{\vec{E}}$ & $x$ & 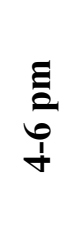 & $\begin{array}{l}\Xi \\
\vdots \\
\infty \\
b\end{array}$ & $\frac{\Xi}{0}$ & $\frac{\Xi}{\grave{2}}$ & 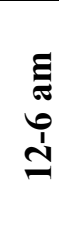 & $\begin{array}{l}\Xi \\
\vdots \\
\infty \\
b \\
0\end{array}$ & 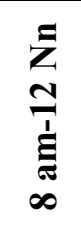 & 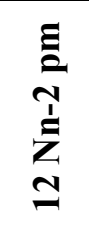 & 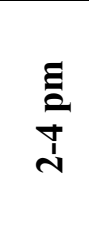 & 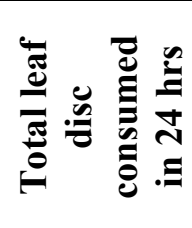 \\
\hline 1 & $\mathrm{H}$ & 1 & 1 & 0.5 & 0.5 & 0.5 & 1 & 1 & 1 & 1 & 8 \\
\hline 2 & $3-\mathrm{Br}$ & 1 & 2 & 0.25 & 1 & 0.1 & 0 & 1 & 1 & 0.5 & 5 \\
\hline 3 & $3-\mathrm{Cl}$ & 0.5 & 0.25 & 0.25 & 0.5 & 0.5 & 0.5 & 1 & 1 & 0.5 & 5 \\
\hline 4 & $4-\mathrm{Cl}$ & 0.5 & 0.5 & 0.25 & 1 & 0.5 & 0.5 & 0.25 & 0.25 & 0.25 & 4 \\
\hline 5 & $3-\mathrm{F}$ & 0.5 & 0.5 & 0.25 & 1 & 0.5 & 0.5 & 0.25 & 0.25 & 0.25 & 4 \\
\hline 6 & 4-F & 0.5 & 0.5 & 0.25 & 1 & 0.5 & 0 & 0 & 0 & 0.25 & 3 \\
\hline 7 & $3-\mathrm{OH}$ & 1 & 2 & 2 & 1 & 0 & 0 & 1 & 1 & 1 & 9 \\
\hline 8 & $2-\mathrm{OCH}_{3}$ & 1 & 1 & 1 & 0.5 & 0.5 & 1 & 2 & 1 & 1 & 10 \\
\hline 9 & $4-\mathrm{OCH}_{3}$ & 1 & 0.5 & 0.5 & 1 & 1 & 0 & 1 & 1 & 1 & 9 \\
\hline 10 & $4-\mathrm{CH}_{3}$ & 0.5 & 1 & 0.5 & 2 & 2 & 0.5 & 0.5 & 1 & 1 & 7 \\
\hline 11 & $3-\mathrm{NO}_{2}$ & 2 & 3 & 3 & 1 & 1 & 1 & 0.5 & 1 & 0 & 12 \\
\hline 12 & $4-\mathrm{NO}_{2}$ & 2 & 3 & 3 & 1 & 0 & 0 & 0.5 & 1 & 0 & 7 \\
\hline
\end{tabular}

Number of leaf discs consumed by the insect (Values are mean + SE of five).

Table 7. Insect antifeedant activity of compound 6 4-fluorostyryl styryl 2-phenothiazenyl ketone at the concentration of 50,100 and $150 \mathrm{ppm}$.

\begin{tabular}{|c|c|c|c|c|c|c|c|c|c|c|}
\hline ב & 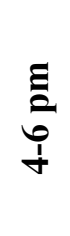 & $\begin{array}{l}\Xi \vdots \\
\infty \\
\vdots \\
b\end{array}$ & $\frac{\Xi}{0}$ & 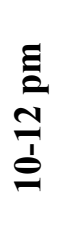 & 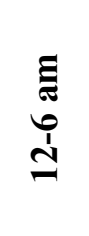 & $\begin{array}{l}\tilde{\sigma} \\
\infty \\
0 \\
b\end{array}$ & 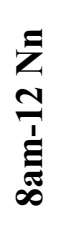 & 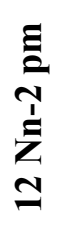 & $\begin{array}{l}\Xi \\
\vdots \\
\dot{a} \\
\sim\end{array}$ & 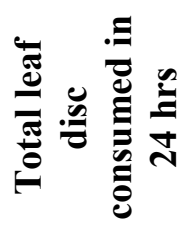 \\
\hline 50 & 0.5 & 0.5 & 0 & 0 & 0 & 0 & 0 & 0 & 0 & 0.1 \\
\hline 100 & 0 & 0.25 & 0 & 0 & 0 & 0 & 0 & 0 & 0 & 0.05 \\
\hline 150 & 0 & 0.5 & .25 & 0 & 0.25 & 0 & 0 & 0 & 0 & 0.1 \\
\hline
\end{tabular}

Number of leaf discs consumed by the insect (Values are mean + SE of five).

The results of the antifeedant activity of keto chalcones presented in (Table 7) and it reveals that the compounds 4-6 are found to reflect remarkable antifeedant among all other chalcones. This test is performed with the insects which took only two-leaf disc soaked under the solution of this compound[20].

Compounds 5 showed enough antifeedant activity but their activities are lesser than 4. Further, compound $\mathbf{4}$ was subjected to measure the antifeedant activity at different 50 , $100,150 \mathrm{ppm}$ concentrations and the observation reveals that as the concentrations are decreases, the activity also decreases.

From the results in Table 8, it is observed that the chalcone $\mathbf{4}$ showed an appreciable antifeedant activity at $150 \mathrm{ppm}$ concentration. 


\section{CONCLUSION}

There are twelve substituted styryl 2-phenothiazenyl ketones have been synthesized by Ground green chemistry method. These chalcones were characterized by their physical constants and spectral data. The electrochemical reduction potential and currents of carbonyl and vinyl parts of these chalcones have been measured. These components were correlated with Hammett substituent constants, F and R parameters. The reduction current of vinyl part and carbonyl carbons were satisfactorily correlated. The multi-regression analysis of these components correlated satisfactorily with Swain-Lupton's, $\mathrm{F}$ and $\mathrm{R}$ parameters. Insect antifeedant activities of these chalcones have been studied using $4^{\text {th }}$ instar larvae Achoea Janata $L$ with castor leaf discs.

\section{Acknowledgement}

The authors thank DST NMR Facility, Department of Chemistry, Annamalai University, Annamalainagar-608002, for recording NMR spectra of all compounds.

\section{References}

[1] Zuman P, Substituent Effects in Organic Polarography, Plenum Press, New-York. 1967.

[2] Boykin D W, Ash M L, Berin F L O, J. Org. Chem. 37(1) (1972) 106-110.

[3] Alston J A, Fry A J, Electrochim. Acta. 49(3) (2004) 455-459.

[4] Morealeda D, Abed D E, Pellissier H, Santelli M, J. Mol. Struct.(TheoChem). 760(1-3) (2006) 113-119.

[5] (a). Arulkumaran R, Vijayakumar S, Sundararajan R, Sakthinathan S P, Kamalakkannan D, Suresh R, Ranganathan K, Vanangamudi G, Thirunarayanan G, Int. Lett. Chem. Phys. Astro. 4 (2012) 17-38.

(b). Sivakumar M, Phrabu sreeneivasan S, Kumar V, Doble M, Bioorg. Med. Chem. Lett. 17(10) (2007) 3169-3172.

[6] Lahtchev K L, Batovska D I, Parushev St P, Ubiyvock V M, Sibirny A A, Eur. J. Med. Chem. 43(10) (2008) 2220-2228.

[7] El-Subbagh Hussein I, Abu-Zaid S M, Mahran M A, Badria Farid A, Al-Obaid A M, J. Med. Chem. 43 (2000) 2915-2920.

[8] Thirunarayanan G, Surya S, Srinivasan S, Vanangamudi G, Sathyendiran V, Spectrochim. Acta Part A. 75 (2010) 152-156.

[9] Deng J, Sanchez T and Lalith Q A M, Bioorg. Med. Chem.15(14), (2007) 4985-5002.

[10] Lin Y M, Zhon Y, Flavin M T, Zhon M, Ne W, Chen F C, Bioorg. Med. Chem. $10(8)(2002) 2795-2802$.

[11] Weber M W, Hunsaker L A, Abcouwer S F, Decker L M, Vander Jagat D L, Bioorg. Med. Chem. 13 (2005) 3811-3820.

[12] Arulkumaran R, Sundararajan R, Vanangamudi G, Subramanian M, Ravi K, Sathiyendiran V, Srinivasan S, Thirunarayanan G, IUP. J. Chem. 3(1) (2010) 82-98.

[13] Thirunarayanan G, Vanangamudi G, Spectrochim. Acta. 81A (2011) 390-396. 
[14] Thirunarayanan G, J. Indian Chem. Soc. 85(4) (2008) 447-451.

[15] Ranganthan K, Arulkumaran K, Kamalakannan D, Vanangamudi G, Thirunarayanan G, Iup. J. Chem. 4(2) (2011) 60-70.

[16] Thirunarayanan G, Vanangamudi G, Sathiyendiran V, Ravi K, Indian J. Chem. 50B(4) (2011) 593-604.

[17] Thirunarayanan G, IUP. J. Chem. 5(1) (2012) 41-53.

[18] Swain C G, Lupton Jr E C, J. Am. Chem. Soc. 90(16) (1968) 4328-4337.

[19] Dethler V G, Chemical insect attractants and repellents, Blackistan, Philadeciphia, 1947.

[20] Thirunarayanan G, J. Saudhi Chem Soc., 2011, doi10.1016/j.jscs.2011.12.003. 